\title{
Magnetic instability in AdS/CFT: Schwinger effect and Euler-Heisenberg Lagrangian of supersymmetric QCD
}

\author{
Koji Hashimoto, ${ }^{a, b}$ Takashi Oka $^{c}$ and Akihiko Sonoda ${ }^{a}$ \\ ${ }^{a}$ Department of Physics, Osaka University, \\ Toyonaka, Osaka 560-0043, Japan \\ ${ }^{b}$ Mathematical Physics Lab., RIKEN Nishina Center, \\ Saitama 351-0198, Japan \\ ${ }^{c}$ Department of Applied Physics, University of Tokyo, \\ Tokyo 113-8656, Japan \\ E-mail: koji@phys.sci.osaka-u.ac.jp, oka@ap.t.u-tokyo.ac.jp, \\ sonoda@het.phys.sci.osaka-u.ac.jp
}

ABSTRACT: To reveal the Schwinger effect for quarks, i.e., pair creation process of quarks and antiquarks, we derive the vacuum decay rate at strong coupling using AdS/CFT correspondence. Magnetic fields, in addition to the electric field responsible for the pair creation, causes prominent effects on the rate, and is important also in experiments such as RHIC/LHC heavy ion collisions. In this paper, through the gravity dual we obtain the full Euler-Heisenberg Lagrangian of $\mathcal{N}=2$ supersymmetric QCD and study the Schwinger mechanism with not only a constant electric field but also a constant magnetic field as external fields. We determine the quark mass and temperature dependence of the Lagrangian. In sharp contrast to the zero magnetic field case, we find that the imaginary part, and thus the vacuum decay rate, diverges in the massless zero-temperature limit. This may be related to a strong instability of the QCD vacuum in strong magnetic fields. The real part of the Lagrangian serves as a generating function for non-linear electro-magnetic responses, and is found such that the Cotton-Mouton effect vanishes. Interestingly, our results of the Schwinger/Cotton-Mouton effects coincide precisely with those of $\mathcal{N}=2$ supersymmetric QED.

KEYwords: Gauge-gravity correspondence, D-branes, Holography and quark-gluon plasmas, QCD

ARXIV EPRINT: 1403.6336 


\section{Contents}

\section{Introduction 1}

2 Euler-Heisenberg Lagrangian with electromagnetic field in massless SQCD

2.1 Full Euler-Heisenberg Lagrangian of strongly coupled $\mathcal{N}=2$ SQCD 4

2.2 Magnetic instability and imaginary part of Lagrangian 6

3 Euler-Heisenberg Lagrangian with electromagnetic field in massive SQCD

3.1 Critical field 9

3.2 Vacuum decay rate in the small mass limit 10

3.3 Nonlinear optical response of the SQCD vacuum: disappearance of the $\begin{array}{ll}\text { Cotton-Mouton effect } & 11\end{array}$

3.4 Coincidence with $\mathcal{N}=2$ supersymmetric QED 12

$\begin{array}{lll}3.4 .1 & \text { Comparison of the vacuum decay rate } & 13\end{array}$

$\begin{array}{ll}\text { 3.4.2 Comparison of the nonlinear optical response coefficient } & 13\end{array}$

$\begin{array}{llr}4 & \text { Summary } & 14\end{array}$

\section{Introduction}

The renowned Schwinger effect, creation process of electron-positron pairs in strong electric fields, is a big challenge in the field of non-linear quantum field theory. Although the Schwinger limit $E \sim m_{e}^{2}$ has not been reached by direct experiments such as strong lasers, similar effective setups in materials are actively investigated. Theoretical foundation of the Schwinger effect $[1]^{1}$ was to evaluate the imaginary part of the effective action of QED under a constant electromagnetic field, the Euler-Heisenberg Lagrangian [4] which dates back to 1936. The Euler-Heisenberg Lagrangian is a generating function of nonlinear electromagnetic responses of the vacuum. In its expression, the electric field couples to the magnetic field in a complicated and nonlinear manner, and the total effective Lagrangian is a starting point in the research of strong fields in QED, including the non-perturbative Schwinger effect.

In [5], two of the present authors derived the Euler-Heisenberg Lagrangian for a supersymmetric QCD in the strong coupling limit using the AdS/CFT correspondence [6-8]. Since the quarks have electric charges, once a strong electric field (not a color electric field ${ }^{2}$

\footnotetext{
${ }^{1}$ For reviews, see $[2,3]$.

${ }^{2}$ For the color electric field and its Schwinger effect, see for example [9-12]. We use the AdS/CFT correspondence, so it is challenging to find how to treat color electromagnetic fields.
} 
but the Maxwell electric field) is applied, a quark antiquark pair is created. The nontrivial part is the gluon interaction at strong coupling in QCD. The quarks are confined, and between the quark and the antiquark a confining force (a QCD string) is present to bind them. If the electric field is strong enough, the quarks are liberated. This truly nonperturbative process is of importance, not only because it can be a realistic phenomenon occurring in the universe, but also because it may be a touchstone for understanding the quark confinement.

There are at least two cases in which the QCD Schwinger effect may play an important role: first, the heavy ion collision experiment, and second, magnetars (neutron stars with a very strong magnetic field). In heavy ion collision experiments, very strong electric fields are generated by the the electric current induced by heavy ions passing by each other. Since the magnetic field is time dependent, there appears strong electric field as well [13-17], and it may be related to the formation of the quark gluon plasma. On the other hand, magnetars are known to be the most dense place in the universe, and the strong magnetic field accompanied by some electric field can occur and affect the core structure of the stars, possibly having a quark phase inside. In these examples, the understanding of QCD and Schwinger effect in strong electric and magnetic fields can be tested by experiments/observations and serves as a playground at which we can test our theoretical knowledge on strongly coupled quantum field theories.

Via the AdS/CFT correspondence, the Schwinger effect of $\mathcal{N}=4$ supersymmetric Yang-Mills theory at strong coupling was calculated in [18], where an explicit saddle point analysis of a quark antiquark pair (a one-instanton calculation) was made. Based on [18], varieties of calculations of the one-instanton amplitude were reported [19-26]. In these papers, a string worldsheet shape in the AdS space was evaluated to calculate the single pair creation. ${ }^{3}$ On the other hand, two of the present authors took a different approach [5] at which a D-brane action in AdS was found to be directly equivalent to the effective Lagrangian (Euler-Heisenberg Lagrangian) including the imaginary part agreeing with instanton summation of the Schwinger effect, for a large electric field. It describes also the real part, and the case where the worldsheet approach breaks down at the large electric field.

The result of [5] is summarized as follows; the Euler-Heisenberg Lagrangian of strongly coupled $\mathcal{N}=2$ supersymmetric QCD at large $N_{c}$ limit was calculated in the presence of a constant electric field using the AdS/CFT correspondence. Its imaginary part explicitly evaluated is found to agree with large electric field expansion of the Schwinger effect of $\mathcal{N}=2$ supersymmetric QED (once the QCD string tension is replaced by the electron mass). However, there, only the electric field was considered. In this paper, we include the full dependence of the magnetic field, which is important as is obvious from the physical situations explained above.

Here we summarize the findings of the present paper:

- We obtain the Euler-Heisenberg Lagrangian of the $\mathcal{N}=2$ supersymmetric QCD in a constant electromagnetic field, at strong coupling and large $N_{c}$ limit.

\footnotetext{
${ }^{3}$ For brane creation approach, see [27]. For an application to EPR, see [28, 29].
} 
- We evaluate the imaginary part of the Euler-Heisenberg Lagrangian, and find that the rate of the quark antiquark creation diverges at zero temperature for massless quarks.

- The divergent rate can be regularized, i.e., the vacuum is unstable but the lifetime becomes finite, by either introducing finite temperature or a quark mass.

- We compute the real part of the Euler-Heisenberg Lagrangian, and show the disappearance of Cotton-Mouton effect in an expansion with the electromagnetic field.

- The imaginary part of the Euler-Heisenberg Lagrangian for a small quark mass is shown to coincide with that of $\mathcal{N}=2$ supersymmetric QED, at the leading order in electron mass. The agreement is found also for the real parts responsible for the Cotton-Mouton effect.

The organization of this paper is as follows. In section 2, using the AdS/CFT correspondence, we obtain the effective action (Euler-Heisenberg Lagrangian) of $\mathcal{N}=2$ massless supersymmetric QCD (SQCD) in a constant electromagnetic field. It is given as a function of the field strengths $\vec{E}$ and $\vec{B}$, the charge density $d$ and the current $j$. For this we use an extension of the dictionary of the AdS/CFT correspondence and the equations of motion. Then we compute the imaginary part of the Euler-Heisenberg Lagrangian for $d=j=0$. We find that the rate of the quark antiquark creation is divergent, and has a regularized form $\log T$ for a small temperature $T$. In section 3, we obtain the Euler-Heisenberg Lagrangian in gapped system when the quark mass is nonzero, and see that the divergent imaginary part is regularized by the small quark mass. We evaluate the real part to study the non-linear electro-magnetic response of the vacuum, and at the third order, we find that the Cotton-Mouton effect disappears. We compare these results with those of weakly coupled supersymmetric QED and find a quantitative agreement, unexpectedly. Finally in section 4 , we summarize our results.

\section{Euler-Heisenberg Lagrangian with electromagnetic field in massless SQCD}

It is a challenging problem to evaluate Schwinger effect in strongly coupled gauge theories. The AdS/CFT correspondence is a universal tool for analyzing such theories. We can consider a classical gravity which is dual to the strongly coupled gauge theories using the AdS/CFT correspondence. In this section, we obtain the Euler-Heisenberg Lagrangian, which is the effective Lagrangian in an external electromagnetic field, for a massless supersymmetric QCD, and evaluate the Lagrangian. First, in section 2.1, we calculate the full Euler-Heisenberg Lagrangian as a function of the electromagnetic fields $\vec{E}$ and $\vec{B}$, the quark number density $d$ and the electric current $j$. Then in section 2.2 , we evaluate the imaginary part and see the divergence due to the presence of the magnetic field. We see that the divergence is regularized by a temperature. 


\subsection{Full Euler-Heisenberg Lagrangian of strongly coupled $\mathcal{N}=2$ SQCD}

Consider the supersymmetric gauge theory on the boundary of an AdS space, which is $\mathcal{N}=4$ supersymmetric Yang-Mills theory with a $\mathcal{N}=2$ hypermultiplet with $\operatorname{SU}\left(N_{c}\right)$ gauge group. The configuration of D-branes realizing the gauge theory is a D3-D7 brane system [30]. Taking a gravity dual, we use an AdS black hole metric as a background metric, in order to see the relation between the rate of quark antiquark creation and a temperature. The probe D7-brane in the AdS space has been well-studied to look at an electric conductivity, that is, an electric current determined by the external electric field [31-33]. ${ }^{4}$ The effective action (the D7-brane action in the AdS) is put to be real to determine the conductivity. Here on the other hand, we are interested in the instability caused by the external electromagnetic field, so the current $j$ is put to zero (or takes some arbitrary value), giving an imaginary part in the effective action: this is how we obtain the Euler-Heisenberg Lagrangian [5].

The AdS black hole metric is the following,

$$
d s^{2}=\frac{R^{2}}{z^{2}}\left[-\left(1-\frac{z^{4}}{z_{H}^{4}}\right) d t^{2}+\left(1-\frac{z^{4}}{z_{H}^{4}}\right)^{-1} d z^{2}+d \vec{x}^{2}\right]+R^{2} d \Omega_{5}^{2},
$$

where the coordinate $z$ is the AdS radial direction, and $z=0$ corresponds to the boundary of the AdS space, and $z=z_{H}$ is the horizon of the black hole. $d \vec{x}^{2}$ is defined by $d \vec{x}^{2}=$ $d x_{1}^{2}+d x_{2}^{2}+d x_{3}^{2} . \quad R$ is the radius of the AdS space. These parameters are given by the following relations between that of the gauge side and that of the gravity side:

$$
z_{H}=\frac{1}{\pi T} \quad R^{4}=2 \lambda \alpha^{\prime 2}
$$

where $\mathrm{T}$ is a temperature, and $\lambda \equiv N_{c} g_{\mathrm{QCD}}^{2}$ is a 't Hooft coupling of the SQCD, and $\alpha$ ' is defined by $\alpha^{\prime}=l_{s}^{2}$.

In the D3-D7 system, the D7-brane has the degree of freedom of the quark in the fundamental representation of the color $\mathrm{SU}\left(N_{c}\right)$ gauge group by coupling to the D3-branes. Going to the gravity dual, the action in the gravity side is a D7-brane action in the AdS space. The D7-brane action is the following:

$$
S_{D 7}=-\mu_{7} \int d t d^{3} \vec{x} d z d^{3} \Omega_{3} \sqrt{-\operatorname{det}\left[P[g]_{a b}+2 \pi \alpha^{\prime} F_{a b}\right]}
$$

where we need not consider the scalar fields on the D7-brane, since we are working with the massless SQCD.

The Euler-Heisenberg effective action is defined by

$$
\mathcal{L}=-i \ln \left\langle e^{-i \int A_{\mu}^{\mathrm{ext}} j^{\mu}}\right\rangle_{0}
$$

which is a function of the external electric and magnetic fields represented by $A_{\mu}^{\text {ext }} \cdot j^{\mu}$ is the $\mathrm{U}(1)$ current operator corresponding to the baryon charge, and the expectation value \langle\rangle$_{0}$ is taken with respect to the "false vacuum", i.e., the vacuum without the field which is now unstable. If the expectation value in (2.4) was taken with the true vacuum, the

\footnotetext{
${ }^{4}$ See also [34-41] for related references.
} 
standard AdS/CFT dictionary [7, 8] states that the effective action is given by the D7-brane action evaluated with the reality condition [31-33] and with the solution of the equation of motion. In ref. [5], two of the present authors proposed that the effective action (2.4) is given as the D7-brane action evaluated with the false-vacuum solution, i.e., the solution of the equation of motion without the electromagnetic fields. Substituting the AdS black hole metric for the D7-brane action, the effective action becomes

$$
\mathcal{L}=-2 \pi^{2} \mu_{7} \int d z \frac{R^{8}}{z^{5}} \sqrt{\xi}
$$

where $d \Omega$-integral is $\operatorname{Vol}\left(S^{3}\right)=2 \pi^{2}$. The factor $\mu_{7}$ is the $\mathrm{D} 7$-brane tension, given by $\mu_{7} \equiv 1 /\left((2 \pi)^{7} g_{s} \alpha^{\prime 4}\right)$. The string coupling constant $g_{s}$ is related to the gauge coupling constant of SQCD as $2 \pi g_{s}=g_{\mathrm{QCD}}^{2}$. Without losing generality, we can choose the direction of the electromagnetic fields. Using the rotation symmetry, we fix the electric field to the $x_{1}$ direction. The magnetic fields are generic in $x_{1}, x_{2}, x_{3}$ directions. Then the $\xi$ in the action is defined by the following:

$$
\begin{aligned}
\xi \equiv & 1-\frac{\left(2 \pi \alpha^{\prime}\right)^{2} z^{4}}{R^{4}}\left[F_{0 z}^{2}+F_{01}^{2} h(z)^{-1}-F_{1 z}^{2} h(z)-F_{12}^{2}-F_{23}^{2}-F_{13}^{2}\right] \\
& -\frac{\left(2 \pi \alpha^{\prime}\right)^{4} z^{8}}{R^{8}}\left[F_{23}^{2}\left\{F_{01}^{2} h(z)^{-1}-F_{1 z}^{2} h(z)\right\}+F_{0 z}^{2}\left\{F_{12}^{2}+F_{23}^{2}+F_{13}^{2}\right\}\right] .
\end{aligned}
$$

The function $h(z)$ is defined as $h(z)=1-z^{4} / z_{H}^{4}$.

Consider rewriting the effective Lagrangian (2.6) in order to see the dependence on the charge density $d$ and the current $j$. We derive the equations of motion from this action. Since we are interested in homogeneous phases, we simply put $\partial_{i}=0(i=1,2,3)$. Then the equations of motion are the following:

$$
\begin{gathered}
\partial_{z}\left[\frac{F_{0 z}}{z \sqrt{\xi}}+\frac{\left(2 \pi \alpha^{\prime}\right)^{2} z^{3}}{R^{4} \sqrt{\xi}} F_{0 z}\left(F_{12}^{2}+F_{23}^{2}+F_{13}^{2}\right)\right]=0 \\
\partial_{0}\left[\frac{F_{0 z}}{z \sqrt{\xi}}+\frac{\left(2 \pi \alpha^{\prime}\right)^{2} z^{3}}{R^{4} \sqrt{\xi}} F_{0 z}\left(F_{12}^{2}+F_{23}^{2}+F_{13}^{2}\right)\right]=0 \\
\partial_{0}\left[\frac{F_{01}}{z \sqrt{\xi}} h(z)^{-1}+\frac{\left(2 \pi \alpha^{\prime}\right)^{2} z^{3}}{R^{4} \sqrt{\xi}} F_{01} F_{23}^{2} h(z)^{-1}\right]+\partial_{z}\left[\frac{F_{1 z}}{z \sqrt{\xi}} h(z)+\frac{\left(2 \pi \alpha^{\prime}\right)^{2} z^{3}}{R^{4} \sqrt{\xi}} F_{1 z} F_{23}^{2} h(z)\right]=0 .
\end{gathered}
$$

In particular, the equations of motions in the case of time-independent field configurations are

$$
\begin{aligned}
\partial_{z}\left[\frac{F_{0 z}}{z \sqrt{\xi}}+\frac{\left(2 \pi \alpha^{\prime}\right)^{2} z^{3}}{R^{4} \sqrt{\xi}} F_{0 z}\left(F_{12}^{2}+F_{23}^{2}+F_{13}^{2}\right)\right] & =0, \\
\partial_{z}\left[\frac{F_{1 z}}{z \sqrt{\xi}} h(z)+\frac{\left(2 \pi \alpha^{\prime}\right)^{2} z^{3}}{R^{4} \sqrt{\xi}} F_{1 z} F_{23}^{2} h(z)\right] & =0 .
\end{aligned}
$$

Next let us evaluate the charge density $d$ and the current $j$ in the gauge theory side. Using the dictionary of the AdS/CFT correspondence, they are respectively

$$
d=\frac{2 \pi \alpha^{\prime} F_{0 z}}{z \sqrt{\xi}}+\frac{\left(2 \pi \alpha^{\prime}\right)^{3} z^{3}}{R^{4} \sqrt{\xi}} F_{0 z}\left(F_{12}^{2}+F_{23}^{2}+F_{13}^{2}\right)
$$




$$
j=\frac{2 \pi \alpha^{\prime} F_{1 z}}{z \sqrt{\xi}} h(z)+\frac{\left(2 \pi \alpha^{\prime}\right)^{3} z^{3}}{R^{4} \sqrt{\xi}} F_{1 z} F_{23}^{2} h(z) .
$$

These charge density and current are substituted into $\xi$, to find

$$
\xi=\frac{1-\frac{\left(2 \pi \alpha^{\prime}\right)^{2} z^{4}}{R^{4}}\left(E_{1}^{2} h(z)^{-1}-\vec{B}^{2}\right)-\frac{\left(2 \pi \alpha^{\prime}\right)^{4} z^{8}}{R^{8}}\left(E_{1} B_{1}\right)^{2} h(z)^{-1}}{1+\frac{z^{6} d^{2}}{R^{4}\left(1+\frac{\left(2 \pi \alpha^{\prime}\right)^{2} z^{4} \vec{B}^{2}}{R^{4}}\right)}-\frac{z^{6} j^{2} h(z)^{-1}}{R^{4}\left(1+\frac{\left(2 \pi \alpha^{\prime}\right)^{2} z^{4} B_{1}^{2}}{R^{4}}\right)}},
$$

where $F_{01} \equiv E_{1}$ is a constant electric field, and $F_{12} \equiv B_{3}, F_{23} \equiv B_{1}, F_{31} \equiv B_{2}$ are constant magnetic fields. Using this $\xi$, the effective Lagrangian with the constant electromagnetic fields is as follows:

$$
\mathcal{L}=-2 \pi^{2} \mu_{7} \int_{0}^{z_{H}} d z \frac{R^{8}}{z^{5}} \sqrt{\frac{1-\frac{\left(2 \pi \alpha^{\prime}\right)^{2} z^{4}}{R^{4}}\left(E_{1}^{2} h(z)^{-1}-\vec{B}^{2}\right)-\frac{\left(2 \pi \alpha^{\prime}\right)^{4} z^{8}}{R^{8}}\left(E_{1} B_{1}\right)^{2} h(z)^{-1}}{1+\frac{z^{6} d^{2}}{R^{4}\left(1+\frac{\left(2 \pi \alpha^{\prime}\right)^{2} z^{4} \vec{B}^{2}}{R^{4}}\right)}-\frac{z^{6} j^{2} h(z)^{-1}}{R^{4}\left(1+\frac{\left(2 \pi \alpha^{\prime}\right)^{2} z^{4} B_{1}^{2}}{R^{4}}\right)}}}
$$

When $j=0$, the solution corresponds to the false vacuum, and (2.15) gives the EulerHeisenberg effective action. This is our result from the AdS/CFT correspondence, and the basis for the following analyses. The imaginary part of the effective action gives half the inverse life time of the false vacuum.

When $j=0$, using the spatial rotation symmetry, we can recover the full $\vec{E}$ and $\vec{B}$ dependence. The Euler-Heisenberg Lagrangian for a generic constant electromagnetic field, at a finite temperature is

$$
\mathcal{L}=-2 \pi^{2} \mu_{7} \int_{0}^{z_{H}} d z \frac{R^{8}}{z^{5}} \sqrt{\frac{1+\beta(z) \vec{B}^{2}-\frac{\beta(z)}{h(z)} \vec{E}^{2}-\frac{\beta(z)^{2}}{h(z)}(\vec{E} \cdot \vec{B})^{2}}{1+\frac{z^{2}}{\left(2 \pi \alpha^{\prime}\right)^{2}} \frac{\beta(z)}{1+\beta(z) \vec{B}^{2}} d^{2}}}
$$

and $\beta(z) \equiv\left(2 \pi \alpha^{\prime}\right)^{2} z^{4} / R^{4}$. In particular, for the vanishing density $d=0$, the EulerHeisenberg Lagrangian is simplified as

$$
\mathcal{L}=-2 \pi^{2} \mu_{7} \int_{0}^{z_{H}} d z \frac{R^{8}}{z^{5}} \sqrt{1+\beta(z) \vec{B}^{2}-\frac{\beta(z)}{h(z)} \vec{E}^{2}-\frac{\beta(z)^{2}}{h(z)}(\vec{E} \cdot \vec{B})^{2}}
$$

In the language of the massless $\mathcal{N}=2 \mathrm{SQCD}$, this Euler-Heisenberg Lagrangian (at a finite temperature and with $d=j=0$ ) is written as

$$
\mathcal{L}=-\frac{N_{c} \lambda}{2^{3} \pi^{4}} \int_{0}^{1 /(\pi T)} \frac{d z}{z^{5}} \sqrt{1+\beta(z) \vec{B}^{2}-\frac{\beta(z)}{h(z)} \vec{E}^{2}-\frac{\beta(z)^{2}}{h(z)}(\vec{E} \cdot \vec{B})^{2}}
$$

where $\beta(z)=\left(2 \pi^{2} / \lambda\right) z^{4}$ and $h(z)=1-(\pi T)^{4} z^{4}$.

\subsection{Magnetic instability and imaginary part of Lagrangian}

In this subsection, we evaluate the imaginary part of the effective Lagrangian, and study the vacuum instability against not only the electric field but also the magnetic field. First, 
the imaginary part at zero temperature $T=0$ and zero quark density diverges: the vacuum is not protected by a gap and thus extremely unstable. In a finite temperature case, the divergence is suppressed. In fact, assuming that the temperature provides a thermal mass for the quarks, the divergence of the imaginary part coincides with the result of a massive SQCD, and further with a supersymmetric QED, as we shall see in the next section.

In the previous subsection, we obtained the effective Lagrangian (2.15) with not only the constant electric field but also the constant magnetic field in the massless system. For simplicity, consider the case when the magnetic field is parallel to the electric field ( $E_{1}$ and $B_{1}$ are nonzero). Then the effective Lagrangian is given by

$$
\mathcal{L}=-2 \pi^{2} \mu_{7} \int_{0}^{z_{H}} d z \frac{R^{8}}{z^{5}} \sqrt{1-\frac{\left(2 \pi \alpha^{\prime}\right)^{2} z^{4}}{R^{4}}\left(E_{1}^{2} h(z)^{-1}-B_{1}^{2}\right)-\frac{\left(2 \pi \alpha^{\prime}\right)^{4} z^{8}}{R^{8}}\left(E_{1} B_{1}\right)^{2} h(z)^{-1}} .
$$

We evaluate the imaginary part of the effective Lagrangian to derive the rate of the quark antiquark creation.

Consider the zero-temperature case, i.e., $z_{H} \rightarrow \infty$. Then the function $h(z)$ approaches unity. The $z$-integral of the imaginary part of the effective Lagrangian is dominated by the third term in the square root of the Lagrangian. Thus, this $z$-integral has a logarithmic divergence. Thus, in the presence of the magnetic field in addition to the electric field, the vacuum decay rate diverges for massless SQCD at strong coupling and at zero temperature. This is in sharp contrast with the zero magnetic field case in which $\operatorname{Im} \mathcal{L}=\frac{N_{c}}{32 \pi} E^{2}$ is obtained [5]. In free Dirac systems, it is known that the divergence of the Euler-Heisenberg effective Lagrangian depends on dimensionality. In the pure electric field case (no magnetic field), for spatial dimension larger than two, the decay rate is finite, while for a $(1+1)$ dimensional system a divergence takes place. Our finding can be understood as an effective dimension reduction by the magnetic field. In a finite magnetic field, Landau levels are formed and the dispersion becomes flat in the two directions perpendicular to the field. Starting from three spatial dimensions, the magnetic field reduces the effective dimension to one. This may explain the divergence we obtain, although, it is unclear if the argument holds for a strongly interacting model.

In a finite temperature system, the divergence of the decay rate is suppressed. In order to evaluate the imaginary part of effective Lagrangian, we change the variable $z$ of this integral to $y$ defined by $y \equiv z / z_{H}$,

$$
\mathcal{L}=-2 \pi^{2} \mu_{7}\left(2 \pi \alpha^{\prime}\right)^{2} R^{4} \chi \int_{0}^{1} \frac{d y}{y^{5}} \sqrt{1-\frac{y^{4}}{\chi}\left(E_{1}^{2}\left(1-y^{4}\right)^{-1}-B_{1}^{2}\right)-\frac{y^{8}}{\chi^{2}}\left(E_{1} B_{1}\right)^{2}\left(1-y^{4}\right)^{-1}},
$$

where $\chi$ is defined as $\chi \equiv R^{4} /\left(2 \pi \alpha^{\prime}\right)^{2} z_{H}^{4}$. As mentioned above, we found that the imaginary part of the Lagrangian diverges in the limit $T \rightarrow 0$. In order to see the dependence on $\chi$ in the square root, we further change the variable $y$ to $Y \equiv \chi^{-\frac{1}{4}} y$,

$$
\mathcal{L}=-2 \pi^{2} \mu_{7}\left(2 \pi \alpha^{\prime}\right)^{2} R^{4} \int_{0}^{\chi^{-\frac{1}{4}}} d Y \frac{\sqrt{1-\left(\chi+E_{1}^{2}-B_{1}^{2}\right) Y^{4}-\chi B_{1}^{2} Y^{8}-\left(E_{1} B_{1}\right)^{2} Y^{8}}}{Y^{5} \sqrt{1-\chi Y^{4}}} .
$$


Let us look for the value of $Y$ at which the integrand turns from real to imaginary. Since $\chi$ is small, we can ignore $\mathcal{O}(\chi)$ term in the numerator, to find the value as $Y=1 / \sqrt{E_{1}}$. So the imaginary part of $\mathcal{L}$ is from the integral over the region $1 / \sqrt{E_{1}}<Y<\chi^{-\frac{1}{4}}$. At the integration, the forth term in the square root of the numerator in the integrand becomes dominant for small $\chi$, hence the imaginary part of the Lagrangian is approximately given by

$$
\operatorname{Im} \mathcal{L} \sim 2 \pi^{2} \mu_{7}\left(2 \pi \alpha^{\prime}\right)^{2} R^{4}\left(E_{1} B_{1}\right) \int_{1 / \sqrt{E_{1}}}^{\chi^{-\frac{1}{4}}} \frac{d Y}{Y \sqrt{1-\chi Y^{4}}} .
$$

Performing the integral for small $\chi$ leads to

$$
\operatorname{Im} \mathcal{L} \sim \frac{N_{c}}{4 \pi^{2}} E_{1} B_{1} \log \frac{1}{T}
$$

where we find a logarithmic dependence on the temperature. ${ }^{5}$

It is straightforward to obtain the Euler-Heisenberg Lagrangian for a generic constant electromagnetic field in finite temperature. For small $T$, we obtain the following dominant term in the divergence,

$$
\operatorname{Im} \mathcal{L}_{T \neq 0}=\frac{N_{c}}{4 \pi^{2}}|\vec{E} \cdot \vec{B}| \log \frac{b(E, B)}{T}+\mathcal{O}\left(T^{0}\right)
$$

Here the constant $b$ is determined as

$$
b(E, B) \equiv\left[\frac{1}{2}\left(\vec{E}^{2}-\vec{B}^{2}+\sqrt{\left(\vec{E}^{2}-\vec{B}^{2}\right)^{2}+4(\vec{E} \cdot \vec{B})^{2}}\right)\right]^{1 / 4}
$$

In addition, in a finite density system, we find a density dependence $(d \neq 0$ but at $T=0)$,

$$
\operatorname{Im} \mathcal{L}_{d \neq 0}=\frac{N_{c}}{4 \pi^{2}}|\vec{E} \cdot \vec{B}| \log \frac{1}{d}+\cdots
$$

This logarithmic dependence is quite similar to the finite temperature case (2.24). In fact, we see in the following that this form is quite common and has a physical interpretation, see (3.15).

\section{Euler-Heisenberg Lagrangian with electromagnetic field in massive SQCD}

In this section, we evaluate the Euler-Heisenberg Lagrangian for the strongly coupled $\mathcal{N}=2$ SQCD with a quark mass in constant electromagnetic fields. We evaluate the imaginary part of the Euler-Heisenberg Lagrangian. The leading term coincides with that of a weakly coupled supersymmetric QED.

\footnotetext{
${ }^{5}$ Divergences appearing in the DBI action and its relation to the validity of the DBI action in the probe limit, see [42].
} 


\subsection{Critical field}

In this section, for simplicity, we consider $T=0$ and $d=0$. First, let us take a D7-brane configuration of the SQCD with vanishing electromagnetic fields. The induced metric on the D7-brane is [30]

$$
d s^{2}=\frac{R^{2}}{z^{2}} \tilde{h}(z)\left(d x^{\mu}\right)^{2}+\frac{R^{2}}{z^{2} \tilde{h}(z)}\left(d z^{2}+d \Omega_{3}^{2}\right),
$$

where $\mu=0,1,2,3$ and $d \Omega_{3}^{2}$ is the metric of a unit 3 -sphere, and

$$
\tilde{h}(z) \equiv 1+\frac{z^{2} \eta^{2}}{R^{2}}
$$

The constant $\eta$ specifies the location of the D7-brane, which is physically related to the quark mass by

$$
\frac{\eta}{2 \pi \alpha^{\prime}}=m_{q}
$$

Turning on the constant electromagnetic fields on the D7-brane, we obtain the EulerHeisenberg Lagrangian for the strongly coupled $\mathcal{N}=2$ SQCD

$$
\mathcal{L}=-2 \pi^{2} \mu_{7} \int_{0}^{\infty} d z \frac{R^{8}}{z^{5}} \sqrt{\xi}
$$

with

$$
\xi \equiv 1+\beta(z) \tilde{h}(z)^{-2}\left(\vec{B}^{2}-\vec{E}^{2}\right)-\beta(z)^{2} \tilde{h}(z)^{-4}(\vec{E} \cdot \vec{B})^{2} .
$$

The $\vec{B}=0$ result agrees with [5], and when $\eta=0$, it reproduces our massless SQCD result $(2.17)$ at $T=0$.

We can evaluate the critical electric field above which the effective Lagrangian acquires an imaginary part. Solving $\xi=0$ for $z$ results in and equation

$$
\tilde{h}(z)^{2}=\beta(z) b^{4}
$$

where the constant $b(E, B)$ is defined in (2.25). This equation is simplified as

$$
1=\left(\frac{2 \pi \alpha^{\prime}}{R^{2}} b^{2}-\frac{\eta^{2}}{R^{4}}\right) z^{2} .
$$

In order for this to have a solution, we need

$$
b(E, B)>\frac{\eta}{\sqrt{2 \pi \alpha^{\prime}} R}=\left(\frac{2 \pi^{2}}{\lambda}\right)^{1 / 4} m_{q} .
$$

This is the condition for having an imaginary part in the Euler-Heisenberg Lagrangian, a signal for vacuum instability. Without the magnetic field $(\vec{B}=0)$, this condition (3.8) reduces to the critical electric field found in [5],

$$
|\vec{E}|>\left(\frac{2 \pi^{2}}{\lambda}\right)^{1 / 2} m_{q}^{2}
$$




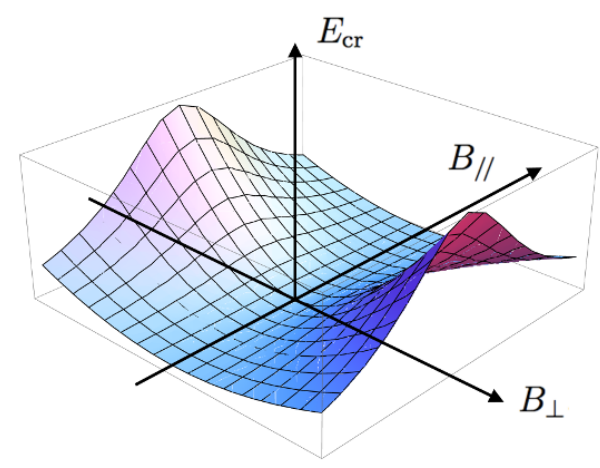

Figure 1. A plot of the critical electric field $E_{\text {cr }}$ as a function of the magnetic field $B_{/ /}$and $B_{\perp}$, for nonzero $m_{q}$. We find that the magnetic field makes the critical electric field larger.

Let us study magnetic field dependence of the critical electric field. The critical electric field $E_{\mathrm{cr}}$ is a solution of the equation

$$
b\left(E_{\mathrm{cr}}, B\right)=\left(\frac{2 \pi^{2}}{\lambda}\right)^{1 / 4} m_{q} .
$$

Decomposing the magnetic field to two components $B_{/ /}$and $B_{\perp}$ (parallel / perpendicular to the electric field), we can plot the value of the critical electric field as a function of the magnetic field $B_{/ /}$and $B_{\perp}$, see figure 1 . We find that the magnetic field does not lower the critical electric field. In fact, the critical electric field is minimized when the perpendicular magnetic field $B_{\perp}$ vanishes, and the minimized value is equal to the critical electric field in the absence of the magnetic field (3.9). In the zero magnetic field case, the critical field coincides with the confining force [5]. This is natural because the vacuum instability takes place when the quark-antiquarks are pulled apart with a force stronger than the confining force. It is strange that the critical field has a $B_{\perp}$ dependence because the gluons mediating the confinement force is not affected by the magnetic field. We leave this puzzle for future consideration.

\subsection{Vacuum decay rate in the small mass limit}

When the quark mass is finite, the vacuum decay rate, i.e., the imaginary part of the effective action, is non-diverging, even above the critical field. Here, we evaluate the small mass asymptotic behavior of the vacuum decay rate. As shown in the previous section, the divergence of the decay rate originates from the integral at large $z$. With non-zero mass represented by the parameter $\eta \neq 0$, the function $\tilde{h}(z)$ has the following $z$ dependence $\left(z_{*} \equiv R^{2} / \eta\right)$

$$
\tilde{h}(z) \sim \begin{cases}1 & \left(z \ll z_{*}\right), \\ \frac{\eta^{2}}{R^{4}} z^{2} & \left(z \gg z_{*}\right) .\end{cases}
$$

This alters the divergent behavior of the integral: for $z \gg z_{*}$, the integrand of the effective Lagrangian behaves as

$$
\frac{R^{8}}{z^{5}} \sqrt{\xi} \sim z^{-5}
$$


whose integral is convergent. The leading behavior of the imaginar part is given by

$$
\operatorname{Im} \mathcal{L} \sim 2 \pi^{2} \mu_{7} \int_{z_{0}}^{z_{*}} d z \frac{R^{8}}{z^{5}} \frac{\left(2 \pi \alpha^{\prime}\right)^{2}}{R^{4}} z^{4}|\vec{E} \cdot \vec{B}|,
$$

where the massless limit corresponds to $z_{*} \rightarrow \infty$. The lower bound of the integral $z_{0}$ is determined by the condition that the Lagrangian becomes imaginary, and is the solution of equation (3.7)

$$
z_{0} \equiv\left(\frac{2 \pi \alpha^{\prime}}{R^{2}} b^{2}-\frac{\eta^{2}}{R^{4}}\right)^{-1 / 2}
$$

The divergence appears when $z_{0} \ll z_{*}$, and the leading term is given by (3.13) which is evaluated as

$$
\begin{aligned}
\operatorname{Im} \mathcal{L} & =\frac{N_{c}}{4 \pi^{2}}|\vec{E} \cdot \vec{B}| \log \frac{z_{*}}{z_{0}}+\cdots \\
& =\frac{N_{c}}{4 \pi^{2}}|\vec{E} \cdot \vec{B}| \log \frac{b(E, B)}{m_{q}}+\text { higher in } \frac{m_{q}}{b(E, B)} .
\end{aligned}
$$

Interestingly, this divergence coincides with (2.24) if we replace the temperature $T$ with the quark mass $m_{q}$. The linear relation between the temperature and the quark mass is commonly found in thermal field theories at weak coupling, and our strongly coupled results are consistent with that. We shall see later that our asymptotic behavior (3.15), agrees with a weak coupling calculation of $\mathcal{N}=2$ supersymmetric QED.

\subsection{Nonlinear optical response of the SQCD vacuum: disappearance of the Cotton-Mouton effect}

The real part of the Euler-Heisenberg Lagrangian has a meaning of electromagnetic vacuum polarization. In particular, if we expand it in terms of the electromagnetic fields, the coefficients gives the non-linear optical response function. As for our system, the first nontrivial term is the fourth order term, which is related to the Kerr effect and the CottonMouton effect, i.e., birefringence induced by magnetic fields (see for a review, [43]). In the following, we study the non-linear optical response below the critical field by evaluating the real part of the Euler-Heisenberg Lagrangian (3.4) as an expansion in terms of the electromagnetic fields.

Defining the Lorentz invariant combinations as

$$
F \equiv \vec{E}^{2}-\vec{B}^{2}, \quad G \equiv \vec{E} \cdot \vec{B},
$$

our Euler-Heisenberg Lagrangian is written as

$$
\mathcal{L}=-2 \pi^{2} \mu_{7} R^{3} \int_{0}^{\infty} d z \frac{R^{5}}{z^{5}} \sqrt{1-g F-g^{2} G^{2}} .
$$

Here we defined

$$
g \equiv \beta(z) \tilde{h}(z)^{-2}=\frac{\left(2 \pi \alpha^{\prime}\right)^{2}}{R^{4}} z^{4}\left(1+\frac{\eta^{2}}{R^{4}} z^{2}\right)^{-2}
$$


If we expand the square root in (3.17) in terms of $F$ and $G^{2}$, we notice that an integral of the form $(n(\geq 1))$

$$
I_{n} \equiv \int_{0}^{\infty} d z \frac{R^{5}}{z^{5}} g(z)^{n}=R\left(2 \pi \alpha^{\prime}\right)^{2} \frac{1}{(1-n)(1-2 n)}\left(\frac{\lambda}{2 \pi^{2} m_{q}^{4}}\right)^{n-1}
$$

determines the expansion coefficients. The Euler-Heisenberg Lagrangian (3.17) is given by

$$
\mathcal{L}=-2 \pi^{2} \mu_{7} R^{3}\left[I_{0}-\frac{1}{2} F I_{1}-\frac{1}{8}\left(F^{2}+4 G^{2}\right) I_{2}+\cdots\right] .
$$

The first term $I_{0}$ is divergent from the first place. It is the vacuum term and we need an appropriate renormalization, as described in [5]. The second term $I_{1}$ corresponds to the charge renormalization, since $F$ is nothing but the original Maxwell electromagnetism Lagrangian. Now, we come to the nontrivial leading correction $I_{2}$. Using the AdS/CFT dictionary for the coefficients, the term with $I_{2}$ is expressed as

$$
\mathcal{L}_{\text {leading }}=\frac{\lambda N_{c}}{3 \cdot 2^{7} \pi^{4} m_{q}^{4}}\left(4 G^{2}+F^{2}\right)
$$

This is the leading electromagnetic correction to the effective action of strongly coupled $\mathcal{N}=2$ supersymmetric QCD.

An interesting observation is that the Cotton-Mouton effect vanishes in (3.21). The response function of Cotton-Mouton effect is given by a combination $c_{0,2}-4 c_{2,0}$ where $c_{0,2}\left(c_{2,0}\right)$ is the coefficient of $G^{2}\left(F^{2}\right)$. We find that

$$
c_{0,2}=4 \frac{\lambda N_{c}}{3 \cdot 2^{7} \pi^{4} m_{q}^{4}}=4 c_{2,0},
$$

so the Cotton-Mouton effect vanishes.

A possible reason for this result would be our supersymmetry (which is not present at low energy in nature), as opposed to the standard QED in which the Cotton-Mouton effect is non-vanishing, that is why experimental confirmation is expected. In fact, if we supersymmetrize the QED, the Cotton-Mouton effect vanishes, as we shall see in the next subsection.

\subsection{Coincidence with $\mathcal{N}=2$ supersymmetric QED}

In this subsection, we compare the Euler-Heisenberg Lagrangian of SQCD with the one-loop result of $\mathcal{N}=2$ supersymmetric QED. A priori, we expect no relation between them because our SQCD is with self-interacting gluons and is evaluated at strong coupling through the AdS/CFT correspondence, while SQED is a weak coupling and photons are not interacting with each other at the one-loop level. However, unexpectedly, we find several coincidences: first is the small electric field asymptotic of the vacuum decay rate, and the second is the leading nonlinear electromagnetic response coefficients. This agreement may be attributed to the supersymmetries. ${ }^{6}$ So, in one aspect, our report here should serve as a consistency check of our calculation of the imaginary D-brane action in the AdS/CFT correspondence.

\footnotetext{
${ }^{6}$ It is known via AdS/CFT correspondence that in SQCD the gluon has a Coulombic potential.
} 


\subsubsection{Comparison of the vacuum decay rate}

First, let us check the divergence in the imaginary part. One-loop QED [11] ${ }^{7}$ has the following expression for the effective Lagrangian when $\vec{E}$ is parallel to $\vec{B}$;

$$
\begin{aligned}
\operatorname{Im} \mathcal{L}_{\text {scalar }} & =\frac{E B}{8 \pi^{2}} \sum_{l=1}^{\infty} \frac{(-1)^{l+1}}{l} \frac{\exp \left[-\pi l m^{2} / E\right]}{2 \sinh (\pi l B / E)}, \\
\operatorname{Im} \mathcal{L}_{\text {spinor }} & =\frac{E B}{8 \pi^{2}} \sum_{l=1}^{\infty} \frac{1}{l} \exp \left[-\pi l m^{2} / E\right] \operatorname{coth}(\pi l B / E) .
\end{aligned}
$$

Here $\mathcal{L}_{\text {scalar }}$ denotes scalar QED (the charged particle is a scalar bosonic field) and $\mathcal{L}_{\text {spinor }}$ is for the ordinary QED. To have $\mathcal{N}=2$ supersymmetry, we need $2 N_{c}$ scalars and $N_{c}$ spinors, and thus

$$
\begin{aligned}
\operatorname{Im} \mathcal{L}_{\mathcal{N}=2 \text { SQED }} & =N_{c}\left(\operatorname{Im} \mathcal{L}_{\text {spinor }}+2 \operatorname{Im} \mathcal{L}_{\text {scalar }}\right) \\
& =\frac{N_{c} E B}{8 \pi^{2}} \sum_{l=1}^{\infty} \frac{1}{l} \exp \left[-\pi l m^{2} / E\right] \frac{\cosh (\pi l B / E)+(-1)^{l+1}}{\sinh (\pi l B / E)}
\end{aligned}
$$

Let us consider a limit of electron mass $m$ going to zero. In the expression above, the factor $\exp \left[-\pi l m^{2} / E\right]$ serves as a cut-off of the summation over $l$. Therefore we can approximate it as

$$
\operatorname{Im} \mathcal{L}_{\mathcal{N}=2 \text { SQED }} \sim \frac{N_{c} E B}{8 \pi^{2}} \sum_{l=1}^{E / \pi m^{2}} \frac{1}{l} \frac{\cosh (\pi l B / E)+(-1)^{l+1}}{\sinh (\pi l B / E)} .
$$

The divergence is due to $\cosh / \sinh \sim 1$ for large $l$, so, for large $E / m^{2}$ we can further approximate it $\operatorname{as}^{8}$

$$
\begin{aligned}
\operatorname{Im} \mathcal{L}_{\mathcal{N}=2 \text { SQED }} & \sim \frac{N_{c} E B}{8 \pi^{2}} \sum_{l=1}^{E / \pi m^{2}} \frac{1}{l} \sim \frac{N_{c} E B}{8 \pi^{2}} \log \frac{E}{\pi m^{2}} \\
& \sim \frac{N_{c}}{4 \pi^{2}} E B \log \frac{\sqrt{E}}{m} .
\end{aligned}
$$

We find that this SQED result is in agreement with our SQCD result (3.15).

\subsubsection{Comparison of the nonlinear optical response coefficient}

Next, we look at the real part when the electromagnetic field is small. We shall see that, again, our SQCD result coincides with that of the SQED.

The integral expression for the Euler-Heisenberg Lagrangian for spinor and scalar QED (for a review, see [2]) is given as

$$
\mathcal{L}_{\text {scalar }}=\frac{1}{16 \pi^{2}} \int_{0}^{\infty} d \eta \frac{e^{-\eta m_{e}^{2}}}{\eta^{3}}\left[\frac{a b \eta^{2}}{\sinh b \eta \sin a \eta}-1+\frac{\eta^{2}}{6}\left(b^{2}-a^{2}\right)\right]
$$

\footnotetext{
${ }^{7}$ See [44-46] for calculations in QED. For supersymmetric calculations, see [47, 48].

${ }^{8}$ In literature, this expression for the dominant imaginary part in QED (non-supersymmetric) can be found in $[49,50]$.
} 


$$
\mathcal{L}_{\text {spinor }}=\frac{-1}{8 \pi^{2}} \int_{0}^{\infty} d \eta \frac{e^{-\eta m_{e}^{2}}}{\eta^{3}}\left[\frac{a b \eta^{2}}{\tanh b \eta \tan a \eta}-1-\frac{\eta^{2}}{3}\left(b^{2}-a^{2}\right)\right] .
$$

Here the real constants $a$ and $b$ are are related to the electro-magnetic fields via $a^{2}-b^{2}=$ $\vec{E}^{2}-\vec{B}^{2}$ and $a b=\vec{E} \cdot \vec{B}$. We can combine the two expressions to obtain the Euler-Heisenberg Lagrangian for the $\mathcal{N}=2$ supersymmetric QED

$$
\begin{aligned}
\mathcal{L}_{\mathcal{N}=2 \text { SQED }} & =N_{c}\left(\mathcal{L}_{\text {spinor }}+2 \mathcal{L}_{\text {scalar }}\right) \\
& =\frac{-N_{c}}{8 \pi^{2}} \int_{0}^{\infty} d \eta \frac{e^{-\eta m_{e}^{2}}}{\eta^{3}}\left[\frac{a b \eta^{2}(\cosh b \eta \cos a \eta-1)}{\sinh b \eta \sin a \eta}-\frac{\eta^{2}}{6}\left(b^{2}-a^{2}\right)\right] .
\end{aligned}
$$

The constant term in the integral of (3.28) and (3.29) correspond to vacuum energy, and cancel each other due to the supersymmetries. We can expand this expression for small $a$ and $b$

$$
\mathcal{L}_{\mathcal{N}=2 \mathrm{SQED}}=\frac{-N_{c}}{8 \pi^{2}} \int_{0}^{\infty} d \eta \frac{e^{-\eta m_{e}^{2}}}{\eta}\left[-\frac{1}{3}\left(a^{2}-b^{2}\right)-\eta^{2} \frac{\left(a^{2}+b^{2}\right)^{2}}{24}+\mathcal{O}\left((a, b)^{6}\right)\right] .
$$

The first term represents charge renormalization and divergent, so here we ignore it. We are interested in the second term, i.e., the leading nontrivial correction to SQED. After the integration over $\eta$, we obtain

$$
\mathcal{L}_{\text {leading }}^{\mathcal{N}=2 \text { SQED }}=\frac{N_{c}}{2^{6} \cdot 3 \pi^{2} m_{e}^{4}}\left(a^{2}+b^{2}\right)^{2}=\frac{N_{c}}{2^{6} \cdot 3 \pi^{2} m_{e}^{4}}\left(4 G^{2}+F^{2}\right)
$$

where $F$ and $G$ are defined in (3.16).

Surprisingly, we find that this SQED result coincides with the SQCD result (3.21). This is obtained if we substitute the following relation

$$
m_{e}^{2} \leftrightarrow \frac{\sqrt{2} \pi}{\sqrt{\lambda}} m_{q}^{2}
$$

The value $\frac{\sqrt{2} \pi}{\sqrt{\lambda}} m_{q}^{2}$ is equal to $E_{\mathrm{cr}}$ which we was found in [5], and this relation was identical to that found in [5] to find an agreement between the imaginary parts of SQED and SQCD for large electric fields.

In summary, in this subsection, we found agreement in asymptotic behaviors of the vacuum decay rate and the nonlinear optical response coefficients between SQCD at the strong coupling and with non-interacting SQED (one loop), assuming a natural relation (3.33).

\section{Summary}

In this work, we studied the response of the vacuum in strong electric and magnetic fields by calculating the Euler-Heisenberg Lagrangian (3.4) of the strongly coupled $\mathcal{N}=2$ supersymmetric QCD in the large $N_{c}$ limit. Above the critical electric field, the vacuum becomes unstable against quark-anti-quark pair production that takes place due to the Schwinger mechanism. The vacuum decay rate is given by the imaginary part of the Euler-Heisenberg 
Lagrangian. The real part is the generating function of response functions of non-linear optical processes. The AdS/CFT correspondence enables us to evaluate the Euler-Heisenberg Lagrangian.

We find that the imaginary part of the effective action diverges when the quark mass (in this theory it is roughly equal to the confinement scale) approaches zero. This divergence appears only in the presence of magnetic fields and we attribute this to the effective dimensional reduction due to Landau quantization of the quarks. We calculated the temperature and quark mass dependence of the decay rate in section 2 and 3 respectively. We found out that the critical electric field depends on the magnetic field (3.8). In our $\mathcal{N}=2$ SQCD, the magnetic field makes the critical electric field larger. We do not fully understand the reason of this magnetic field dependence.

We also found, in some limits, the Euler-Heisenberg Lagrangian of large $N_{c}$ SQCD in the strong coupling limit agrees with non-interacting $\mathcal{N}=2$ supersymmetric QED. The imaginary part in strong electromagnetic field, and the other is the real part in the weak field expansion, at fourth order. This is an interesting coincidence which we did not expect: the background electromagnetic field breaks the supersymmetry so this coincidence cannot be explained by it. Although we do not understand this, our finding may serve as an evidence that the AdS/CFT dictionary can be extended to the false vacuum. This encourages us to further investigate non-supersymmetric setup in the AdS/CFT correspondence at which we can evaluate the Euler-Heisenberg Lagrangian and the Schwinger effect at more realistic strongly coupled field theories. We shall report this elsewhere [51].

\section{Acknowledgments}

K.H. would like to thank Y. Hidaka. We are grateful to the hospitality of OIST. K.H. and T.O. are supported by KAKENHI (Grant No. 23654096, 23740260, 24224009). This research was partially supported by the RIKEN iTHES project.

Open Access. This article is distributed under the terms of the Creative Commons Attribution License (CC-BY 4.0), which permits any use, distribution and reproduction in any medium, provided the original author(s) and source are credited.

\section{References}

[1] J.S. Schwinger, On gauge invariance and vacuum polarization, Phys. Rev. 82 (1951) 664 [INSPIRE].

[2] G.V. Dunne, Heisenberg-Euler effective Lagrangians: basics and extensions, in From fields to strings, vol. 1, M. Shifman et al. eds., World Scientific, Singapore (2005), pg. 445 [hep-th/0406216] [INSPIRE].

[3] W. Dittrich and H. Gies, Probing the quantum vacuum, Springer-Verlag, Berlin Germany (2000).

[4] W. Heisenberg and H. Euler, Consequences of Dirac's theory of positrons, Z. Phys. 98 (1936) 714 [physics/0605038] [INSPIRE]. 
[5] K. Hashimoto and T. Oka, Vacuum instability in electric fields via AdS/CFT: Euler-Heisenberg Lagrangian and Planckian thermalization, JHEP 10 (2013) 116 [arXiv: 1307.7423] [INSPIRE].

[6] J.M. Maldacena, The large- $N$ limit of superconformal field theories and supergravity, Adv. Theor. Math. Phys. 2 (1998) 231 [Int. J. Theor. Phys. 38 (1999) 1113] [hep-th/9711200] [INSPIRE].

[7] S.S. Gubser, I.R. Klebanov and A.M. Polyakov, Gauge theory correlators from noncritical string theory, Phys. Lett. B 428 (1998) 105 [hep-th/9802109] [InSPIRE].

[8] E. Witten, Anti-de Sitter space and holography, Adv. Theor. Math. Phys. 2 (1998) 253 [hep-th/9802150] [INSPIRE].

[9] A. Yildiz and P.H. Cox, Vacuum behavior in quantum chromodynamics, Phys. Rev. D 21 (1980) 1095 [INSPIRE].

[10] J. Ambjørn and R.J. Hughes, Canonical quantization in non-Abelian background fields. 1, Annals Phys. 145 (1983) 340 [inSPIRE].

[11] N. Tanji, Dynamical view of pair creation in uniform electric and magnetic fields, Annals Phys. 324 (2009) 1691 [arXiv:0810.4429] [INSPIRE].

[12] N. Tanji and K. Itakura, Schwinger mechanism enhanced by the Nielsen-Olesen instability, Phys. Lett. B 713 (2012) 117 [arXiv:1111.6772] [InSPIRE].

[13] D.E. Kharzeev, L.D. McLerran and H.J. Warringa, The effects of topological charge change in heavy ion collisions: 'event by event P and CP-violation', Nucl. Phys. A 803 (2008) 227 [arXiv:0711.0950] [INSPIRE].

[14] V. Skokov, A.Y. Illarionov and V. Toneev, Estimate of the magnetic field strength in heavy-ion collisions, Int. J. Mod. Phys. A 24 (2009) 5925 [arXiv:0907.1396] [inSPIRE].

[15] V. Voronyuk et al., (Electro-)magnetic field evolution in relativistic heavy-ion collisions, Phys. Rev. C 83 (2011) 054911 [arXiv:1103.4239] [INSPIRE].

[16] A. Bzdak and V. Skokov, Event-by-event fluctuations of magnetic and electric fields in heavy ion collisions, Phys. Lett. B 710 (2012) 171 [arXiv:1111.1949] [INSPIRE].

[17] W.-T. Deng and X.-G. Huang, Event-by-event generation of electromagnetic fields in heavy-ion collisions, Phys. Rev. C 85 (2012) 044907 [arXiv: 1201.5108] [INSPIRE].

[18] G.W. Semenoff and K. Zarembo, Holographic Schwinger effect, Phys. Rev. Lett. 107 (2011) 171601 [arXiv: 1109.2920] [INSPIRE].

[19] J. Ambjørn and Y. Makeenko, Remarks on holographic Wilson loops and the Schwinger effect, Phys. Rev. D 85 (2012) 061901 [arXiv:1112.5606] [INSPIRE].

[20] S. Bolognesi, F. Kiefer and E. Rabinovici, Comments on critical electric and magnetic fields from holography, JHEP 01 (2013) 174 [arXiv:1210.4170] [INSPIRE].

[21] Y. Sato and K. Yoshida, Holographic description of the Schwinger effect in electric and magnetic fields, JHEP 04 (2013) 111 [arXiv: 1303.0112] [INSPIRE].

[22] Y. Sato and K. Yoshida, Potential analysis in holographic Schwinger effect, JHEP 08 (2013) 002 [arXiv: 1304.7917] [INSPIRE].

[23] Y. Sato and K. Yoshida, Holographic Schwinger effect in confining phase, JHEP 09 (2013) 134 [arXiv:1306.5512] [INSPIRE]. 
[24] Y. Sato and K. Yoshida, Universal aspects of holographic Schwinger effect in general backgrounds, JHEP 12 (2013) 051 [arXiv:1309.4629] [INSPIRE].

[25] D. Kawai, Y. Sato and K. Yoshida, The Schwinger pair production rate in confining theories via holography, Phys. Rev. D 89 (2014) 101901 [arXiv:1312.4341] [INSPIRE].

[26] M. Sakaguchi, H. Shin and K. Yoshida, No pair production of open strings in a plane-wave background, arXiv:1402.2048 [INSPIRE].

[27] A.S. Gorsky, K.A. Saraikin and K.G. Selivanov, Schwinger type processes via branes and their gravity duals, Nucl. Phys. B 628 (2002) 270 [hep-th/0110178] [INSPIRE].

[28] J. Sonner, Holographic Schwinger effect and the geometry of entanglement, Phys. Rev. Lett. 111 (2013) 211603 [arXiv:1307.6850] [INSPIRE].

[29] M. Chernicoff, A. Güijosa and J.F. Pedraza, Holographic EPR pairs, wormholes and radiation, JHEP 10 (2013) 211 [arXiv:1308.3695] [INSPIRE].

[30] A. Karch and E. Katz, Adding flavor to AdS/CFT, JHEP 06 (2002) 043 [hep-th/0205236] [INSPIRE].

[31] A. Karch and A. O'Bannon, Metallic AdS/CFT, JHEP 09 (2007) 024 [arXiv:0705.3870] [INSPIRE].

[32] A. Karch and A. O'Bannon, Holographic thermodynamics at finite baryon density: some exact results, JHEP 11 (2007) 074 [arXiv:0709.0570] [INSPIRE].

[33] J. Erdmenger, R. Meyer and J.P. Shock, AdS/CFT with flavour in electric and magnetic Kalb-Ramond fields, JHEP 12 (2007) 091 [arXiv:0709.1551] [INSPIRE].

[34] S.A. Hartnoll and C.P. Herzog, Ohm's law at strong coupling: $S$ duality and the cyclotron resonance, Phys. Rev. D 76 (2007) 106012 [arXiv:0706.3228] [INSPIRE].

[35] T. Albash, V.G. Filev, C.V. Johnson and A. Kundu, Finite temperature large- $N$ gauge theory with quarks in an external magnetic field, JHEP 07 (2008) 080 [arXiv: 0709.1547] [INSPIRE].

[36] T. Albash, V.G. Filev, C.V. Johnson and A. Kundu, Quarks in an external electric field in finite temperature large- $N$ gauge theory, JHEP 08 (2008) 092 [arXiv:0709.1554] [INSPIRE].

[37] C.V. Johnson and A. Kundu, External fields and chiral symmetry breaking in the Sakai-Sugimoto model, JHEP 12 (2008) 053 [arXiv:0803.0038] [INSPIRE].

[38] O. Bergman, G. Lifschytz and M. Lippert, Response of holographic QCD to electric and magnetic fields, JHEP 05 (2008) 007 [arXiv:0802.3720] [INSPIRE].

[39] K.-Y. Kim, S.-J. Sin and I. Zahed, Dense and hot holographic QCD: finite baryonic E field, JHEP 07 (2008) 096 [arXiv:0803.0318] [INSPIRE].

[40] N. Evans, A. Gebauer and K.-Y. Kim, $E, B, \mu, T$ phase structure of the D3/D7 holographic dual, JHEP 05 (2011) 067 [arXiv:1103.5627] [INSPIRE].

[41] M.S. Alam, V.S. Kaplunovsky and A. Kundu, Chiral symmetry breaking and external fields in the Kuperstein-Sonnenschein model, JHEP 04 (2012) 111 [arXiv:1202.3488] [INSPIRE].

[42] F. Bigazzi, A.L. Cotrone and J. Tarrio, Charged D3/D7 plasmas: novel solutions, extremality and stability issues, JHEP 07 (2013) 074 [arXiv: 1304.4802] [INSPIRE].

[43] R. Battesti and C. Rizzo, Magnetic and electric properties of quantum vacuum, Rept. Prog. Phys. 76 (2013) 016401 [arXiv:1211.1933] [INSPIRE]. 
[44] V.I. Ritus, Method of eigenfunctions and mass operator in quantum electrodynamics of a constant field, Sov. Phys. JETP 48 (1978) 788 [Zh. Eksp. Teor. Fiz. 75 (1978) 1560] [INSPIRE].

[45] S.L. Lebedev and V.I. Ritus, Virial representation of the imaginary part of the Lagrange function of the electromagnetic field, Sov. Phys. JETP 59 (1984) 237 [Zh. Eksp. Teor. Fiz. 86 (1984) 408] [INSPIRE].

[46] I.K. Affleck, O. Alvarez and N.S. Manton, Pair production at strong coupling in weak external fields, Nucl. Phys. B 197 (1982) 509 [inSPIRE].

[47] I.L. Buchbinder, S.M. Kuzenko and A.A. Tseytlin, On low-energy effective actions in $N=2$, $N=4$ superconformal theories in four-dimensions, Phys. Rev. D 62 (2000) 045001 [hep-th/9911221] [INSPIRE].

[48] S.M. Kuzenko and I.N. McArthur, Low-energy dynamics in $N=2$ super QED: two loop approximation, JHEP 10 (2003) 029 [hep-th/0308136] [INSPIRE].

[49] Y. Hidaka, T. Iritani and H. Suganuma, Fast vacuum decay into quark pairs in strong color electric and magnetic fields, AIP Conf. Proc. 1388 (2011) 516 [arXiv:1103.3097] [InSPIRE].

[50] Y. Hidaka, T. Iritani and H. Suganuma, Fast vacuum decay into particle pairs in strong electric and magnetic fields, arXiv:1102.0050 [INSPIRE].

[51] K. Hashimoto, T. Oka and A. Sonoda, work in progress. 